\title{
Arthroskopische Chirurgie an der Schulter
}

\author{
— Martin Jaeger, Peter Ogon, Wolfgang Köstler, Norbert P. Südkamp
}

\section{Zusammenfassung}

Die Schulterarthroskopie stellt eine moderne Behandlungsoption zur Therapie von verschiedenen Schulterverletzungen und -erkrankungen dar. Bewährt hat sich die arthroskopische Stabilisierung von Schulterinstabilitäten. Typischerweise lässt sich der traumatisch abgerissene, ventrale Kapsel-Labrum-Komplex mit Fadenankern gut refixieren. Je nach Laxität des Schultergelenkes kann der Eingriff mit einem Kapselshift kombiniert werden. Dabei kann die arthroskopische Stabilisierung ähnlich gute Ergebnisse hinsichtlich einer RezidivLuxationsrate von $5-10 \%$ aufweisen, wie sie vom bisherigen Goldstandard nach offenen Stabilisierungsverfahren bekannt ist. Weitere Indikationen ergeben sich im Rahmen der arthroskopischen Refixation von Rupturen der Rotatorenmanschette, v.a. der Supraund Infraspinatussehne. In der Hand des Geübten lassen sich auch Rupturen der Subskapularissehne gut rekonstruieren. Vorteilhaft hat sich darüber hinaus die arthroskopische Kalkausräumung im Rahmen eines chronisch rezidivierenden Verlaufes der Tendinosis calcarea herausgestellt. Auch lassen sich Infektionen des Glenohumeralgelenkes in den Stadien I-III nach Gächter mit guten Ergebnissen arthroskopisch behandeln.

\section{Arthroscopic Surgery of the Shoulder}

Arthroscopy of the shoulder represents a modern treatment option for various injuries and diseases of the shoulder. The arthroscopic stabilisation of shoulder instabilities is now a proven method. Typically a traumatically separated ventral capsule-labrum complex can be fixed well with suture anchors. Depending on the laxity of the shoulder joint the intervention can be combined with a capsule shift. In this way arthroscopic stabilisation can give similarly good results with a recurrence of $5-10 \%$ as the previous gold standard of open stabilisation methods. Further indications for shoulder arthroscopy are the arthroscopic refixation of rotator cuft tears, in particular, the supra- and infraspinatus tendons. With sufficient experience it is also possible to reconstruct ruptures of the subscapularis tendon. In addition, arthroscopic removal of calcific deposits in cases of chronic recurrent calcifying tendinitis has proved advantageous. Furthermore, infections of the glenohumeral joint in stages I - III according to Gächter can be treated with good results by arthroscopy.

\section{Einleitung}

Mithilfe der Arthroskopie ergeben sich umfangreiche Möglichkeiten in Diagnostik und Therapie zahlreicher Gelenkerkrankungen und -verletzungen.

Dabei haben sich seit ihren Anfängen nicht zuletzt aufgrund einer steten technischen Weiterentwicklung ihre Indikationen und Möglichkeiten permanent weiter ausgedehnt. Im Bereich der Schulterarthroskopie erweisen sich insbesondere folgende Therapiefelder als vorteilhaft und dankbar:
- Arthroskopische Stabilisierung von Schulterinstabilitäten

- Arthroskopische Refixation von Rupturen der Rotatorenmanschette

- Arthroskopische Ausräumung von Kalkdepots

- Arthroskopische Therapie von Schultergelenksinfektionen.

\section{Equipment}

Voraussetzung für eine effektive Schulterarthroskopie ist ein modernes Equipment. Bewährt hat sich ein ArthroskopieTurm bestehend aus folgenden Komponenten:

- digitale, mittlerweile autoklavierbare Kameraeinheit

- Lichtquelle

- Rollenpumpeneinheit mit kontrollierter Drucksteuerung

- arthroskopisches Motorsystem

- System zur Elektrochirurgie (z.B. OPES Orthopaedic Procedure Electrosurgical System by Arthrex ${ }^{\circledR}$ )

Darüber hinaus wird eine digitale Dokumentationseinheit benötigt, die das Speichern von Bildern, sowie Videosequenzen

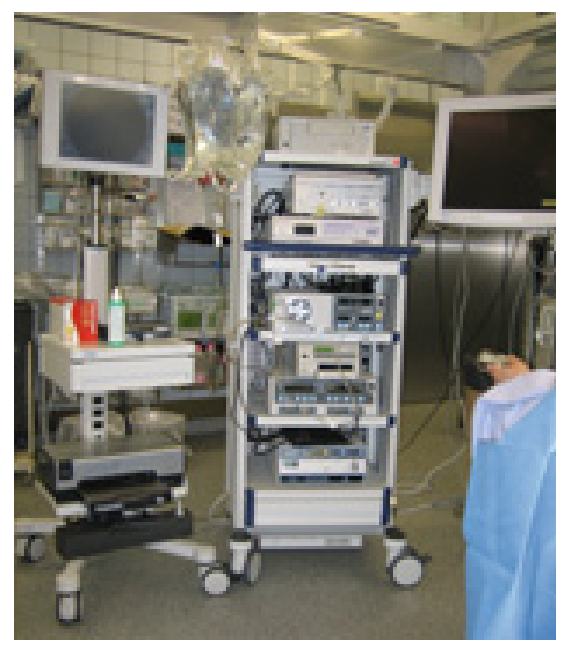

Abb. 1 Arthroskopie-Turm mit Dokumentationseinheit. 
erlaubt (Abb.1). Diese Daten werden vorteilhaft auf einem Server in einem LAN abgelegt und hier regelmäßig mit einer speziellen Back-up-Software gesichert.

\section{Lagerung}

Zur Schulterarthroskopie eignen sich prinzipiell die beach-chair- und die Seitenlagerung. Von uns wird die Seitenlagerung favorisiert, da sie eine bequeme Aufhängung des zu operierenden Armes und damit eine gute Übersicht des OPGebietes ermöglicht und zusätzlich eine sichere Platzierung der vielen Kabel, respektive Schläuche garantiert. Hierzu lagern wir den Patienten auf der kontralateralen Seite auf einer Vakuummatratze. Zur Druckentlastung der unten liegenden Schulter wird eine Auflagefläche im Bereich des proximalen Thorax einmodelliert. Das Ohr auf der zu operierenden Seite schützen wir während der Operation z. B. beim Ablegen von Instrumenten durch Auflage eines kleinen, flachen Gelkissens. Zur Abdeckung haben sich konfektionierte Einmal-Sets bewährt. Der zu operierende Arm wird in einem Doppelarmhalter mit 6 Kilogramm Längszug und bei arthroskopischen Stabilisierungen mit 2 Kilogramm Vertikalzug unter sterilen Bedingungen aufgehängt. Wir drehen den OP-Tisch mit dem Kopf in den Saal, um nicht nur von dorsal, sondern auch von der Stirnseite operieren zu können (Abb.2).

Die Beach-chair-Lagerung benutzen wir nur noch selten bei Patienten, die mit einer hohen Wahrscheinlichkeit einer Konversion zu einem offenen Verfahren operiert werden, um dadurch Zeiten durch Umlagerung einzusparen.

\section{Arthroskopische Stabilisierung von Schulterinstabilitäten}

\section{Operative Therapie der traumatischen Erstluxation}

Das operative Vorgehen richtet sich nach der ihr zugrunde liegenden Pathologie. Diese sollte gezielt und wenn möglich anatomisch repariert werden. Darüber hinaus sind Kenntnisse über den Spontanverlauf sowie insbesondere über epidemiologische Daten notwendig, um den Patienten umfassend beraten zu können. Operationsindikationen sind der Tab. 1 zu entnehmen. Ein unmittelbares, operatives Vorgehen im Sinne einer NotfallOperation ist im Rahmen einer traumatischen Erstluxation arthroskopisch nicht

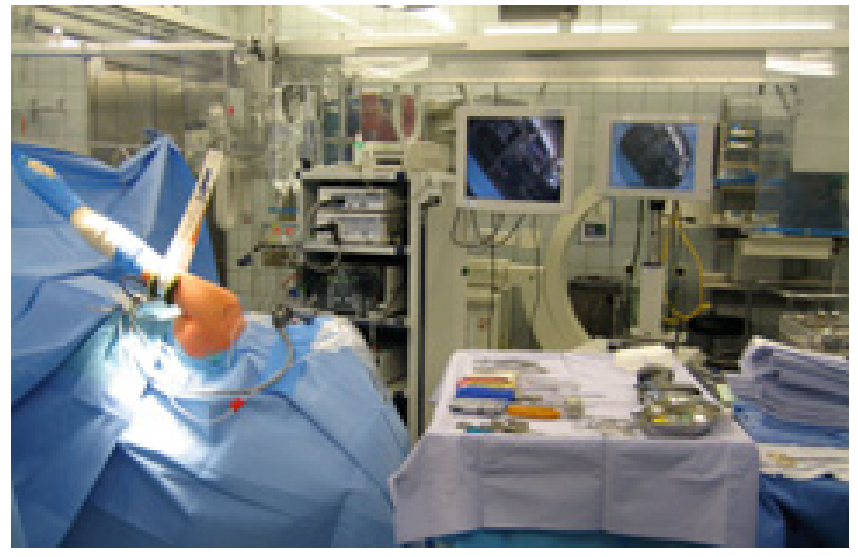

Abb. 2 Seitenlagerung zur Schulterarthroskopie. gegeben. Der Zeitpunkt kann elektiv gewählt werden.

Besonders gute Ergebnisse sind bei der Therapie der traumatischen Erstluxation des jungen Patienten zu erwarten. Ein adäquates Trauma eine Fremdreposition unter Analgosedierung in der Anamnese, der radiologische Nachweis einer HillSachs-Läsion, sowie der klinische Ausschluss einer Hyperlaxität selektieren dieses in der Regel hoch motivierte und durch eine gute Compliance gekennzeichnete Patientengut. Hier ist u.E. die arthroskopische Therapie schon nach der Erstluxation gerechtfertigt und besonders geeignet. Arthroskopisch lässt sich die Pathologie genau evaluieren sowie sicher und kontrolliert reparieren.
Die arthroskopische Stabilisierung kann dabei ähnlich gute Ergebnisse hinsichtlich einer Rezidiv-Luxationsrate von $5-10 \%$ erreichen, wie sie vom bisherigen Goldstandard nach offenen Stabilisierungsverfahren bekannt ist [1-8] (Tab.2). Jedoch scheint sie schneller durchführbar und mit einer geringeren Zugangsmorbidität versehen. Insbesondere die Ablösung der Subscapularissehne verbunden mit unterschiedlichen Shift-Techniken bei ihrer Refixierung, die bei allen offenen Verfahren notwendig ist, scheint im Vergleich neben einer stärkeren postoperativen Einschränkung des Schulterbewegungsumfanges auch mit einer höheren Rate von bis zu 23\% Subscapularis-Insuffizienzen einherzugehen $[1,9]$.

Tab. 1 Operationsindikationen im Rahmen einer traumatischen glenohumeralen Erstluxation

\section{Absolute Operationsindikationen}

Irreponible Luxation mit interponiertem Weichteilgewebe

Knöcherne Bankart-Läsionen jenseits des kritischen Wertes von 1/5 der kaudalen Glenoidfläche (d. h. größer als $15 \mathrm{~mm}$ Länge und $5 \mathrm{~mm}$ Breite)

Mehr als $5 \mathrm{~mm}$ dislozierte Fraktur des Tuberkulum majus

Begleitende Ruptur der Subskapularissehne

Begleitende Rotatorenmanschettenruptur beim älteren Patienten

Begleitende instabile Mehrfragment-Fraktur des Humeruskopfes im Sinne einer Luxationsfraktur

\section{Relative Operationsindikationen}

Alter zwischen 16 und 30 Jahre

Hoher Funktionsanspruch

Adäquates Trauma ohne Selbstreposition

Bankart- und Hill-Sachs-Läsion

Ausschluss einer Hyperlaxität

Gute Compliance 
Tab. 2 Rezidivraten nach arthroskopischer posttraumatischer ventraler Schulterstabilisierung mit Fadenankern

\begin{tabular}{|c|c|c|c|c|c|c|}
\hline Autor & Jahr & Studiendesign & Follow-up & Patienten & Technik & Rezidive \\
\hline Bottoni [1] & 2006 & $\begin{array}{l}\text { Prospektiv } \\
\text { randomisiert }\end{array}$ & 32 Mon. & $\begin{array}{l}29 \\
32\end{array}$ & $\begin{array}{l}\text { Offen vs. } \\
\text { Arthrosk. (Fadenanker) }\end{array}$ & $\begin{array}{l}0 \% \\
0 \%\end{array}$ \\
\hline Carreira [5] & 2006 & Prospektiv & 48 Mon. & 85 & Arthrosk. (Fadenanker) & $5 \%$ \\
\hline Marquardt [6] & 2006 & Prospektiv & 42 Mon. & 54 & Arthrosk. (Fadenanker) & $7,5 \%$ \\
\hline Tjoumakaris [2] & 2006 & Retrospektiv & $\begin{array}{l}56 \text { Mon. } \\
40 \text { Mon. }\end{array}$ & $\begin{array}{l}24 \\
69\end{array}$ & $\begin{array}{l}\text { Offen vs. } \\
\text { Arthrosk. (Fadenanker) }\end{array}$ & $\begin{array}{l}4 \% \\
1 \%\end{array}$ \\
\hline Fabbriciani [7] & 2004 & $\begin{array}{l}\text { Prospektiv } \\
\text { randomisiert }\end{array}$ & 24 Mon. & $\begin{array}{l}30 \\
30\end{array}$ & $\begin{array}{l}\text { Offen vs. } \\
\text { Arthrosk. (Fadenanker) }\end{array}$ & $\begin{array}{l}0 \% \\
0 \%\end{array}$ \\
\hline Ide [4] & 2004 & Prospektiv & 42 Mon. & 55 & Arthrosk. (Fadenanker) & $7 \%$ \\
\hline Kim [8] & 2002 & Prospektiv & 39 Mon. & $\begin{array}{l}30 \\
59\end{array}$ & $\begin{array}{l}\text { Offen vs. } \\
\text { Arthrosk. (Fadenanker) }\end{array}$ & $\begin{array}{l}6,7 \% \\
3,4 \%\end{array}$ \\
\hline
\end{tabular}

Bildgebend fordern wir eine Röntgendarstellung der Schulter in 2 Ebenen, um Glenoid- oder Humeruskopffrakturen auszuschließen. Mit der MRT lassen sich präoperativ die verschiedenen LabrumPathologien, v.a. die Bankart- und die Perthes-Läsion diagnostizieren (Abb. 3). Insbesondere bei älteren Patienten (über 40 Jahre) lassen sich in Abhängigkeit vom Alter des Patienten auch zunehmend Rupturen der Rotatorenmanschette feststellen.

Weitere Spektren der arthroskopischen Schulterstabilisierung finden sich bei der Rezidivinstabilität [10], der dorsalen Instabilität sowie bei Verletzungen des Bizepssehnenankers (SLAP-Läsionen).

Zur arthroskopischen Stabilisierung des abgerissenen Kapsel-Labrum-Komplexes stehen dem Operateur verschiedene Verfahren zur Auswahl. Zur Fixierung haben sich vor allem Nahtanker bewährt. Die transglenoidale Nahttechnik nach Caspari sowie die Verwendung von Staples sind nur noch selten zu beobachten.

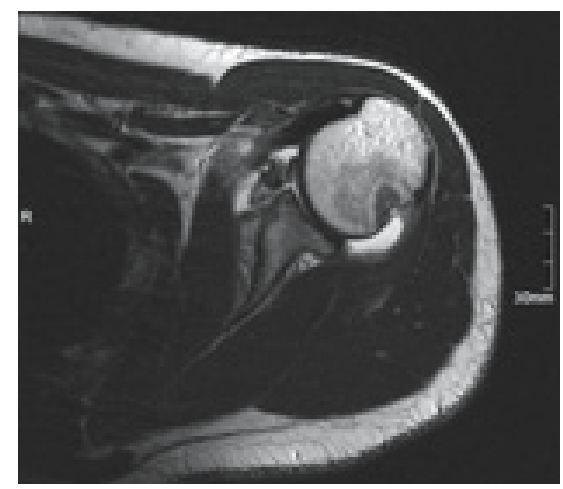

Abb. 3 Darstellung einer Bankart-Läsion im MRT.

\section{Fixierung des ventral abgerissenen Kapsel-Labrum-Komplexes mit Naht- ankern}

Nach Abschluss der diagnostischen Arthroskopie des glenohumeralen $\mathrm{Ge}$ lenkes über einen typischen dorsalen Standard-Zugang werden unter Sicht zunächst ein $8,25 \mathrm{~mm}$ ventraler Standard-Zugang unmittelbar kranial der Subskapularissehne sowie ein $5,75 \mathrm{~mm}$ anteroventraler Zugang dorsal der langen Bizepssehne angelegt. Die Insertion von transparenten Zugangskanülen mit Gewinde zur Prophylaxe einer Dislokation haben sich zum einen zum Schutz des Weichteilgewebes, zum anderen zur Vermeidung von Gewebebrücken bewährt. Es erfolgt zunächst die Mobilisation des ventralen Labrums, bis selbiges von der darunter liegenden Muskulatur gelöst ist und nach Ablassen des Spüldruckes spontan auf Glenoidhöhe aufschwimmt. Hierbei bewährt sich insbesondere bei einem weit medial im Sinne einer ALPSA-Läsion fehlverheilten Labrum das Wechseln der Optik in das

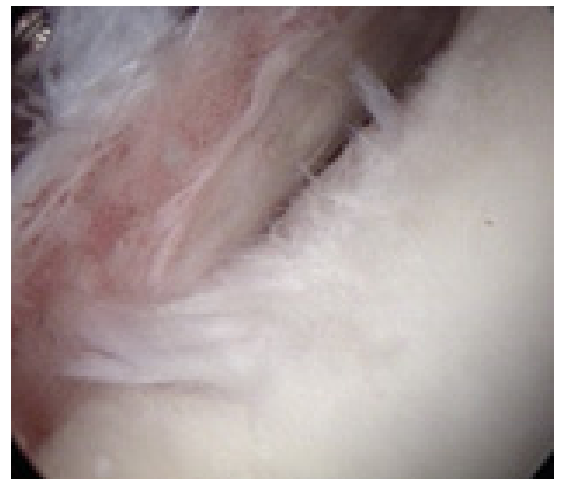

Abb. 4 Arthroskopische Darstellung einer Bankart-Läsion. anteroventrale Portal, um auch hier die Operationsschritte kontrolliert unter Sicht durchzuführen. Je nach Laxität des Schultergelenkes kann zusätzlich noch ein arthroskopischer inferiorer Kapselshift erforderlich sein. Anschließend wird der Glenoidhals von anhängendem Weichteilgewebe debridiert. Hierzu eignen sich unterschiedlich gebogene Bankart-Raspeln, aber auch Weichteilshaver. Sinnvoll ist es ferner, den ventralen Anteil des Gelenkknorpels sparsam mit einer arthroskopischen Kürette zu debridieren, um hier eine gute Einheilung des Labrums zu ermöglichen. Zur Fixierung des mobilisierten Kapsel-Labrum-Komplexes eignen sich Nähte, die in der Lasso-Technik platziert werden. Diese werden temporär über den anteroventralen Zugang geborgen und mit dem Lasso anschließend im ventralen Zugang ausgeleitet. Die Fixierung kann mit speziellen, fadenlosen Ösen-Ankern erfolgen, die knotenfrei einen hohen Anpressdruck ermöglichen. Weiter kranial können zusätzlich Naht-Anker zur Anwendung kommen (Abb.4 und 5).

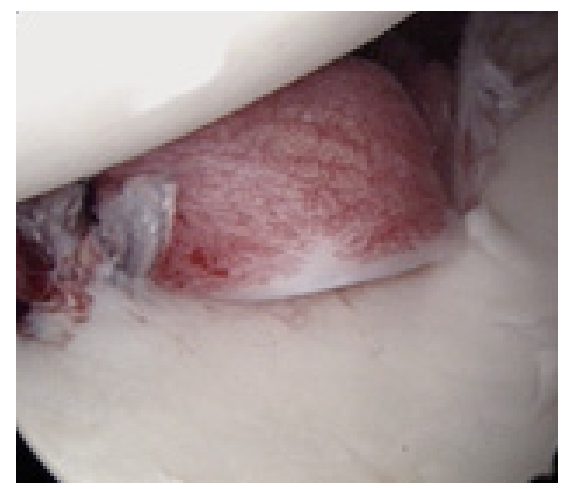

Abb.5 Z.n. arthroskopischer Refixation einer Bankart-Läsion mit BioPushLock ${ }^{\circledR}$ und BioFASTak ${ }^{\circledR}$-Ankern. 
Sollte während der Operation der Arbeitsraum eng und unübersichtlich sein, hilft es, die Lagerung zu kontrollieren. Ein nach ventral subluxierter Humeruskopf sollte nach dorsal reponiert werden. Ebenso sollte der Vertikal-Zug den proximalen Humerus nach lateral und kranial ziehen. Hierzu ist eine zum Oberarm senkrechte Zugrichtung der Schlinge bei ausreichend weit ausgezogenem Armausleger notwendig.

Nur ein komplett mobilisierter KapselLabrum-Komplex lässt sich regelrecht am Glenoid refixieren. Zur Kontrolle eignet sich das Ablassen des Spüldruckes, um zu beobachten, wie das Labrum auf Glenoidhöhe aufschwimmt

\section{Nachbehandlung}

Zur Nachbehandlung wird die operierte Schulter tagsüber für vier Wochen in einer Schulterorthese (z.B. Omomed ${ }^{\circledR}$ ) immobilisiert, um die Bewegungen in der anfangs nicht erlaubten Abduktion und Anteversion über $90^{\circ}$ zu erschweren. Die Außenrotation ist in dieser Zeit vollständig untersagt. Nachts wird die Schulter z.B. in der medi Arm fix ${ }^{\circledR}$ Bandage ruhig gestellt. Bereits am 1. postoperativen Tag wird mit einer krankengymnastischen Nachbehandlung begonnen, die die o.g. Bewegungseinschränkungen berücksichtigt. Vor Wiederaufnahme eines sportlichen Trainings, in der Regel nach 3-6 Monaten, muss das Muskelaufbautraining abgeschlossen sein. Hierzu sollte insbesondere bei aktiven Sportlern der Trainingszustand mit einem Messverfahren (z.B. Cybex ${ }^{\circledR}$ ) überprüft werden. Unseres Erachtens hat es sich bewährt, die Patienten rechtzeitig im Rahmen der Nachbehandlung anzuleiten und ihnen, sowie dem weiterbehandelnden Hausarzt und dem weiterbehandelnden Krankengymnasten, ein standardisiertes Nachbehandlungsschema an die Hand zu geben.

\section{Arthroskopische Refixation von Rupturen der Rotatorenmanschette}

Eine weitere Domäne der arthroskopischen Schulterchirurgie stellt die Refixation von Rupturen der Rotatorenmanschette dar. Bewährt hat sich die Einteilung der kompletten Rupturen nach Bateman [11] (Tab.3) sowie der Retraktionsgrad nach Patte [12] (Tab.4). Partielle Rupturen werden bevorzugt nach Ellman [13] (Tab. 5) klassifiziert. Besonders die Supra- und Infraspinatussehnenrupturen mit geringer oder mäßiger
Atrophie der Muskulatur eignen sich gut. Die Subskapularis-Sehnenrupturen lassen sich ebenfalls arthroskopisch refixieren, sind aber technisch deutlich anspruchsvoller. Kontrovers werden die Teilrupturen der Rotatorenmanschette diskutiert. Insbesondere bei artikularseitigen Teilrupturen der Supraspinatussehne scheint mitunter eine alleinige subakromiale Dekompression nicht ausreichend. Wir empfehlen hier die Refixation der Sehne.

Entscheidend für den Therapieerfolg ist unter anderem die breitflächige Reinsertion auf dem entsprechend vorbereiteten Areal, dem sogenannten Footprint. Hierzu haben sich verschiedene Naht- und Fixationstechniken bewährt. Im Vergleich bietet die Doppel-Reihen-Fixierung mit Faden-Ankern die besten Ergebnisse. [14]. Darüber hinaus ist die Qualität der Sehne von entscheidender Bedeutung. Während frische Rupturen dem Nahtmaterial genügend suffizientes Material als Widerlager geben, zeigen über lange Zeit bestehende, chronische und degenerativ veränderte Sehnen wesentlich schlechtere Ergebnisse. Hier zeigt sich, dass eine subtile Mobilisation der Sehne sowohl

Tab. 3 Klassifkation der Rotatorenmanschettenruptur nach Bateman [11]

\begin{tabular}{lll} 
Grad & Größe & $\begin{array}{l}\text { Bezeich- } \\
\text { nung }\end{array}$ \\
\hline 1 & $<1 \mathrm{~cm}$ & Klein \\
2 & $1-3 \mathrm{~cm}$ & Mittel \\
3 & $3-5 \mathrm{~cm}$ & Groß \\
4 & $>5 \mathrm{~cm}$ & Massiv
\end{tabular}

subakromial als auch subtendinös in der Lage ist, die Spannung der Sehne nach Fixation deutlich zu reduzieren. Diese Mobilisierung ist im Vergleich zu einem offenen Vorgehen aufgrund der besseren Visualisierung und in Verbindung mit einem $\mathrm{OPES}^{{ }^{\circledR}}{ }_{-}$Gerät und damit verbundenen geringeren Blutungsneigung ausgesprochen effektiv.

\section{Operationstechnik der arthroskopischen Refixation einer Supraspinatus/Infraspi- natus-Sehnenruptur}

In Seitenlagerung erfolgt zunächst eine diagnostische Arthroskopie des Glenohumeralgelenkes. Nach Ausschluss einer behandelbaren Pathologie erfolgt das Eingehen in den subakromialen Raum. Über einen lateralen Standard-Zugang erfolgt zunächst eine subakromiale Dekompression mit elektrischen Rotationsmessern und Fräsen. Eine Durchtrennung des Ligamentum coracoacromiale ist nicht notwendig. Es kann z.B. mit einem Elektrohaken von der Unterseite des Akromions abgeschoben werden. In der Folge wird die Ruptur sauber dargestellt und die Manschette mit dem OPES $^{\circledR}$-Gerät sowohl subakromial als auch subtendinös mobilisiert. Nach Umsetzen der Optik in einen zusätzlich geschaffenen, posterolateralen Zugang wird der Foot-print debridiert und für die Refixation vorbereitet. Insbesondere bei einer Doppel-Reihen-Fixierung ist auf eine genügend nach lateral vorgenommene Mobilisation des M. deltoideus zu achten. Über eine gesonderte laterale Inzision nahe dem Akromion werden anschließend in der Regel zwei doppelt beladene Faden-Anker der me-

Tab. 4 Sehnenretraktion der Rotatorenmanschettenruptur nach Patte [12]

\begin{tabular}{ll} 
Sektor & Beschreibung \\
\hline I & Sehnenstumpf liegt zwischen Tuberkulum majus und Apex \\
II & Sehnenstumpf liegt zwischen Apex und Glenoid \\
III & Sehnenstumpf liegt hinter dem Glenoid
\end{tabular}

Tab.5 Klassifikation der partiellen Rotatorenmanschettenruptur nach Ellman [13]
Lokalisation
A
B
artikular- oder gelenkseitig
bursa- oder akromionseitig
C
intratendinös
Größe (Defektfläche Breite in mm x max. Retraktion in mm)
Grad 1
$<3 \mathrm{~mm}$ tief
Grad 2
3-6 mm tief
Grad 3
$>6 \mathrm{~mm}$ tief 
dialen Reihe senkrecht in das Tuberkulum majus inseriert. Ihre Fäden lassen sich mit verschiedenen Techniken durch die Rotatorenmanschette führen. Bewährt hat sich dabei die Verwendung einer Scorpion ${ }^{\circledR}$-Zange, mit der die Fäden im Sinne einer U-Naht jeweils durch die Manschette gestoßen werden können. Um subakromiale Verknotungen oder Fadenüberwerfungen zu vermeiden, ist es vorteilhaft, die durchstoßenen Fäden durch das dorsale Portal auszuleiten. Empfehlenswert ist die Verwendung von zwei transparenten $5,75 \mathrm{~mm}$ Schleusen im lateralen und dorsalen Portal, um das Weichteilgewebe zu schonen und Gewebebrücken zu vermeiden. Nachdem derartig die Rotatorenmanschette je nach Größe der Ruptur mit ein bis vier U-Nähten durchstoßen wurde, werden die Fäden verknotet. Dabei bewährt sich, während des Knotens die Manschette mit noch nicht geknoteten Fäden über das laterale Portal zu reponieren und die Knotung selbst von dorsal vorzunehmen, um derartig in Repositionsrichtung die Knoten zu schieben. Die verknoteten Fäden selbst werden im Anschluss in einer zweiten, weiter lateral platzierten Reihe fixiert. Dabei lässt sich insbesondere mit fadenlosem Ösen-Anker ein hoher Anpressdruck der Manschette auf dem Foot-print ermöglichen. Je nach Anzahl der verwendeten Ösen-Anker entsteht durch die aufgespannten Fäden die Form eines „V“ oder eines „W“ (Abb.6).

Große Rupturen weisen regelhaft einen mobileren Schenkel auf, der im Sinne eines L-Shifts häufig aus dem Infraspinatus heraus mobilisiert werden kann. Der dadurch entstehende Längsriss kann mit einer intertendinösen Naht verschlossen werden.

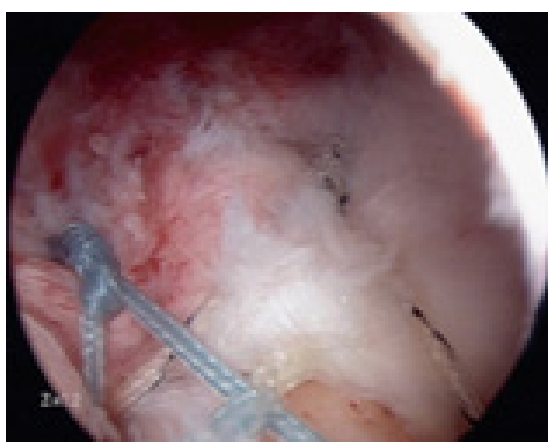

Abb. 6 Arthroskopische Refixation einer Supraspinatus-Sehnenruptur in der Suturebridge-Technik.
Im Rahmen der arthroskopischen Refixation von Rupturen der Rotatorenmanschette ist ein konsequentes Fadenmanagement besonders wichtig. Übersicht ist dabei essenziell. Bewährt hat sich die Verwendung von Schleusen, um Gewebebrücken zu vermeiden. Durchstoßene Fäden können ferner temporär gut durch andere Portale, z. B. posteriores Standardportal, ausgeleitet werden, um sie aus dem Sichtfeld zu nehmen.

\section{Nachbehandlung}

Postoperativ wird die operierte Schulter, je nach Spannung der Naht, auf einem $15^{\circ}$ oder $30^{\circ}$ Schulterabduktionskissen für 6 Wochen immobilisiert. In dieser Zeit sind eine aktive glenohumerale Bewegung sowie eine passive Adduktion untersagt. Nach sechs Wochen geben wir nach sonographischer Kontrolle des Operationsergebnisses die aktive Bewegung in allen Ebenen frei.

\section{Arthroskopische Kalkausräumung}

Patienten, die an einem chronisch rezidivierenden Krankheitsverlauf der rendinosis calcarea leiden, der trotz intensiven konservativen Behandlungsversuchen keine Besserungstendenz zeigte, profitieren besonders von einer arthroskopischen Kalkausräumung. Wichtig erscheint hier, präoperativ eine exakte Lokalisationsbestimmung des oder der Kalkdepots vorzunehmen. Bewährt hat sich hierbei die sonographische Beurteilung der Kalkdepots in der Technik nach Ogon [22]. Mit ihr lassen sich die Kalkdepots vier Quadranten zuordnen, die senkrecht zur lateralen Akromionkante eingeteilt werden. Derartig ist es möglich, dass zum einen nur eine partielle subakromiale Bursektomie vorgenommen werden muss und die Kalkdepots selbst deutlich schneller und zielsicherer gefunden werden können. Eine Markierung der Kalkdepots mit Fäden oder eine intraoperative Röntgendiagnostik ist damit unserer Erfahrung nach nicht notwendig. Unterschiedlich diskutiert wird, inwieweit eine komplette Ausräumung des Kalkdepots u.U. mit Einsatz von Weichteilshavern notwendig ist. Diese kann insofern aufwendig sein, als manchmal die Wandung eines Kalkdepots fest mit der Manschette verbunden ist und die Sehnenfasern selbst verkalkt sind. Es scheint jedoch so zu sein, dass in diesen Fällen eine komplette Ausräumung nicht notwendig ist und sich restliche Kalkstrukturen nach arthroskopischer Ausräumung im Verlauf weiter auflösen und regelhaft symptomlos sind. Eine großflächige iatrogene Beschädigung der Rotatorenmanschette ist daher nicht gerechtfertigt.

\section{Operationstechnik der arthroskopischen Kalkausräumung}

In Seitenlagerung werden nach Aufhängen des zu operierenden Armes zunächst die anatomischen Landmarken und Portale sowie die Quadranten nach Ogon angezeichnet [22] (Abb. 7). Nach Ausschluss einer intraartikulären Pathologie im Rahmen einer diagnostischen Arthroskopie des Glenohumeralgelenkes erfolgt über einen lateralen Zugang eine partielle Bursektomie im mutmaßlichen Quadranten. Mit einer Injektionskanüle wird das Kalkdepot durch Nadelung im zuvor diagnostizierten Quadranten lokalisiert und in seiner Größenausdehnung bestimmt (Abb.8). Das Kalkdepot selbst wird mit einem kleinen Tasthaken eröffnet und der Kalk zusammen mit der Spülflüssigkeit ausgerührt, respektive ausgespült. Typischerweise resultiert hierbei das Bild eines „Schneegestöbers“, dass durch viele kleine Kalkpartikel entsteht, die ausgespült werden (Abb.9).

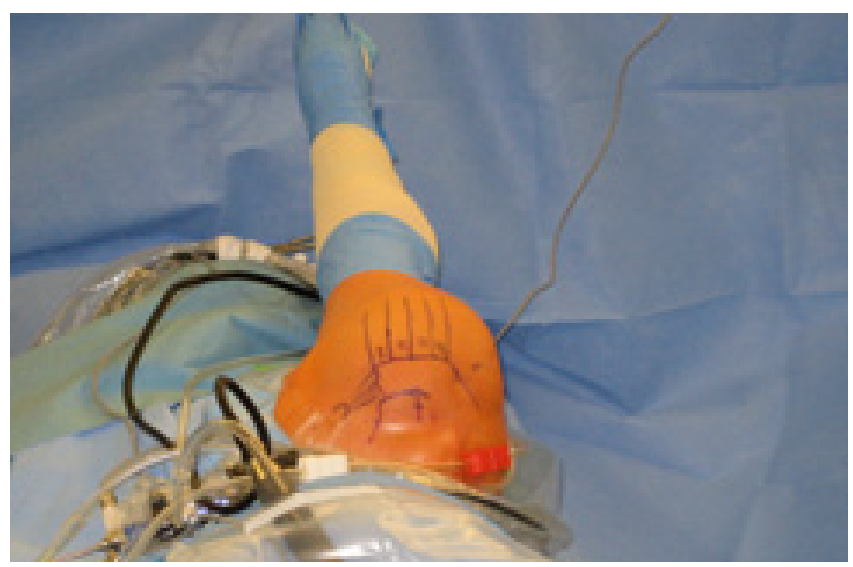

Abb. 7 Seitenlagerung und Zeichnung der Quadranten nach Ogon [22]. 


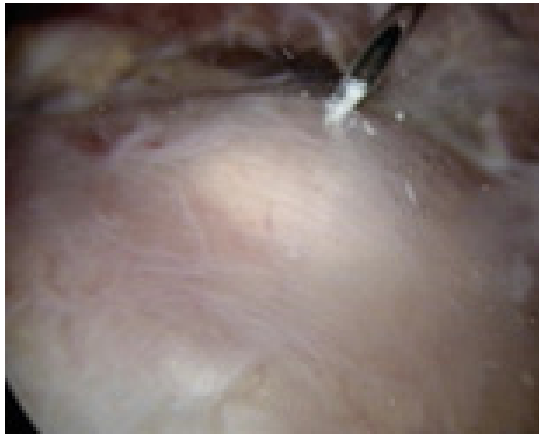

Abb. 8 Arthroskopische Darstellung eines sich deutlich vorwölbenden Kalkdepots ansatznah im 2. Quadranten. Größenbestimmung mit einer Kanüle.

Mitunter lassen sich auch größere, pastöse Kalkstränge exprimieren, die ausgesaugt werden können. Abschließend erfolgt eine Therapiekontrolle durch erneutes Nadeln mit einer Injektionskanüle. Sollten dabei noch verbliebene Kalkareale lokalisiert werden, werden diese über eine weitere Inzision mit dem Tasthaken ausgeräumt.

In der Operationstechnik nach Ogon ist es wichtig, darauf zu achten, dass sich der abduziert aufgehängte Arm in Neutralstellung befindet. Nur so kann eine exakte Korrelation zur präoperativen, sonographischen Diagnostik und Quadranteneinteilung hergestellt werden.

\section{Nachbehandlung}

Postoperativ werden die operierten Schultern schmerzadaptiert für ein bis drei Tage in einem Gilchrist-Verband immobilisiert. Es folgt eine schmerzadaptierte frühfunktionelle Nachbehandlung.

\section{Arthroskopische Infektbehandlung des Schultergelenkes}

Eine Infektion des Schultergelenkes ist glücklicherweise ein seltenes Ereignis. Ihre Rate liegt nach Schulterarthroskopien bei $0,71 \%$ [23], teilweise bedingt durch die enormen Mengen an Spülflüssigkeit. Das Infektionsrisiko steigt mit zunehmender Operationsdauer, Anzahl der vorhergehenden Eingriffen, Ausmaß des operativen Vorgehens, sowie insbesondere nach einer vorhergehenden Steroidinjektion [24]. Wichtig erscheint, den Schultergelenksinfekt rasch zu diagnostizieren und unverzüglich $\mathrm{zu}$ therapieren. Dabei stellen sowohl die Diagnostik als auch die Therapie eine Notfallindikation dar und erlauben keine Verzögerung. Denn nur eine frühzeitig eingeleitete und aggressive Therapie hat

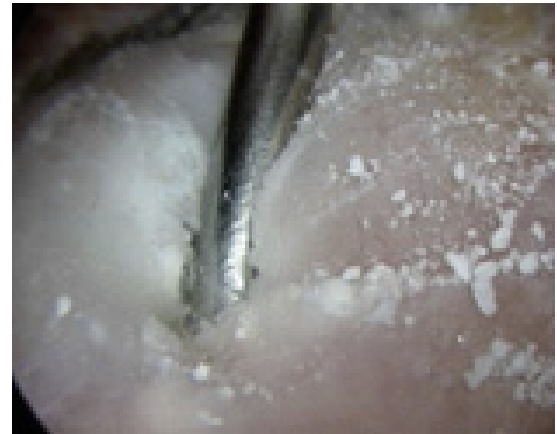

Abb.9 Arthroskopische Ausräumung eines Kalkdepots im 2. Quadranten. Bild eines "Schneegestöbers“.

gute Aussicht für einen Therapieerfolg. Als sehr nützlich hat sich in der frühen Phase der Diagnostik sowie zur Therapiebeurteilung im Verlauf die Bestimmung des C-reaktiven Proteins (CRP) erwiesen, dass als „Akut-Phase-Protein“ sehr sensitiv reagiert. Nach Gächter [25] lassen sich Gelenkinfektionen in vier Sta- dien einteilen, von denen sich eine stadienorientierte Therapie ableiten lässt (Tab. 6 und 7). Insbesondere die Stadien I bis III nach Gächter sind in einem besonderen Maß einer arthroskopischen Therapie zugänglich. Mit ihr lassen sich alle Gelenkkompartimente kontrolliert lavagieren und eine arthroskopische Synovektomie sehr sorgfältig minimalinvasiv durchführen (Abb.10). Durch das geringe zugangsbedingte Operationstrauma ist die Morbidität nicht erhöht. Begleitet wird die arthroskopische Therapie von einer initial intravenösen, Erreger-adaptierten Antibiose, die nach Normalisierung der serologischen Entzündungsparameter noch vier Wochen weitergeführt werden sollte [26]. Da bei der arthroskopischen Stabilisierung und der arthroskopischen Manschettenrefixation Fremdmaterialien eingebracht werden, die wenn irgend möglich im Rahmen der operativen Therapie erhalten werden sollen, empfiehlt sich aufgrund des typischen Erregerspektrums

Tab. 6 Klassifikation von Gelenkinfekten nach Gächter [25]

\begin{tabular}{ll} 
Stadium & Beschreibung \\
\hline I & $\begin{array}{l}\text { Noch durchsichtige Gelenkflüssigkeit, Rötung der Synovialmembran, evtl. pe- } \\
\text { techiale Einblutung, keine radiologischen Veränderungen }\end{array}$ \\
\hline II & $\begin{array}{l}\text { Ausgeprägte inflammatorische Reaktion der Synovialmembran, fibrinöse Be- } \\
\text { lege, eitrige Gelenkflüssigkeit, keine radiologischen Veränderungen }\end{array}$ \\
\hline III & $\begin{array}{l}\text { Verdickung der Synovialmembran, Kompartmentbildung in verschiedenen } \\
\text { Gelenkabschnitten, keine radiologischen Veränderungen }\end{array}$ \\
\hline IV & $\begin{array}{l}\text { Aggressiver Panus mit Infiltrationen des Gelenkknorpels, evtl. Unterminierung } \\
\text { des Gelenkknorpels, radiologische Zeichen der subchondralen Osteolyse, evtl. } \\
\text { ossäre Erosion und Zystenbildung }\end{array}$
\end{tabular}

Tab. 7 Stadienorientierte operative Therapie von Gelenkinfekten nach Gächter [25]

\begin{tabular}{|c|c|}
\hline Stadium & Beschreibung \\
\hline I & $\begin{array}{l}\text { Arthroskopische ausgedehnte Lavage aller Gelenkkompartimente mit syste- } \\
\text { mischer Antibiotikatherapie; bei anhaltenden klinischen und blutchemischen } \\
\text { Zeichen eines Infekts Rearthroskopie innerhalb weniger Tage }\end{array}$ \\
\hline II & $\begin{array}{l}\text { Arthroskopische Lavage aller Gelenkkompartimente mit Entfernung aller fibri- } \\
\text { nöser Belege und Entfernung von Eiterherden im Bereich der Synovia ohne } \\
\text { komplette Synovektomie; sorgfältiges Monitoring der postoperativen kli- } \\
\text { nischen und blutchemischen Entzündungsparameter, evtl. repititive Re- } \\
\text { arthroskopie innerhalb weniger Tage }\end{array}$ \\
\hline III & $\begin{array}{l}\text { Arthroskopische Lavage aller Gelenkkompartimente mit Entfernung aller fibri- } \\
\text { nöser Belege und Entfernung von Eiterherden im Bereich der Synovia ohne } \\
\text { komplette Synovektomie; sorgfältiges Monitoring der postoperativen kli- } \\
\text { nischen und blutchemischen Entzündungsparameter, evtl. repititive Re- } \\
\text { arthroskopie innerhalb weniger Tage }\end{array}$ \\
\hline IV & $\begin{array}{l}\text { Hier ist ein rein arthroskopisches Vorgehen nur in seltenen Fällen und bei } \\
\text { erheblicher Erfahrung des Operateurs indiziert; neben den unter Stadium III } \\
\text { ergriffenen Maßnahmen muss gleichzeitig ein sorgfältiges Debridement der } \\
\text { avitalen Knorpelbereiche sowie der betroffenen Knochenanteile erfolgen. Dies } \\
\text { ist in vielen Fällen nur in offener Technik möglich. }\end{array}$ \\
\hline
\end{tabular}




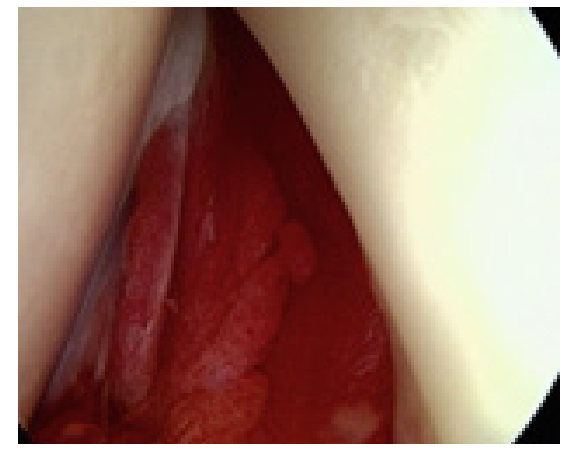

Abb. 10 Intraartikulärer Befund einer glenohumeralen Infektion Stadium Gächter I.

eine Kombinationstherapie mit Rifampicin.

Hierbei handelt es sich um ein potentes Antibiotikum, dass eine bakterizide Wirkung auf oberflächenadhäsive, langsam wachsende und Biofilm produzierende Bakterien hat. Aufgrund der raschen Resistenzentwicklung sollte Rifampicin jedoch immer in Kombination mit einem Penicillin oder Cephallosporin gegeben werden.

Die Diagnostik eines Schultergelenkinfektes stellt ebenso wie seine Therapie eine Notfallindikation dar. Ein kompromissloses, rasches und stadiengerechtes Vorgehen ist Voraussetzung für ein gutes Behandlungsergebnis

\section{Literatur}

1 Bottoni LTCCR, Smith MAJEL, Berkowitz MAJMJ, Towle CDRRB, Moore COLJH. Arthroscopic Versus Open Shoulder Stabilization for Recurrent Anterior Instability: A Prospective Randomized Clinical Trial. The American journal of sports medicine 2006; 34: 17301737

2 Tjoumakaris FPMD, Abboud JAMD, Hasan SAMD, Ramsey MLMD, Williams GRMD. Arthroscopic and Open Bankart Repairs Provide Similar Outcomes. Clinical orthopaedics and related research 2006; 446: 227-232

3 Mazzocca AD, Brown FM, Jr., Carreira DS, Hayden J, Romeo AA. Arthroscopic Anterior Shoulder Stabilization of Collision and Contact Athletes. The American journal of sports medicine 2005; 33: 52-60
${ }^{4}$ Ide J, Maeda S, Takagi K. Arthroscopic Bankart Repair Using Suture Anchors in Athletes: Patient Selection and Postoperative Sports Activity. The American journal of sports medicine 2004; 32: 1899-1905

5 Carreira DS, Mazzocca AD, Oryhon J, et al. A Prospective Outcome Evaluation of Arthroscopic Bankart Repairs: Minimum 2-Year Follow-up. The American journal of sports medicine 2006; 34: 771-777

${ }^{6}$ Marquardt B, Witt K-A, Liem D, Steinbeck J, Potzl W. Arthroscopic Bankart Repair in Traumatic Anterior Shoulder Instability Using a Suture Anchor Technique. Arthroscopy: The Journal of Arthroscopic \& Related Surgery 2006; 22: 931-936

${ }^{7}$ Fabbriciani C, Milano G, Demontis A, et al. Arthroscopic versus open treatment of Bankart lesion of the shoulder: A prospective randomized study. Arthroscopy: The Journal of Arthroscopic \& Related Surgery 2004; 20: 456-462

8 Kim S-H, Ha K-I, Kim S-H. Bankart repair in traumatic anterior shoulder instability. Arthroscopy: The Journal of Arthroscopic \& Related Surgery 2002; 18: 755-763

${ }^{9}$ Sachs RA, Williams B, Stone ML, Paxton L, Kuney M. Open Bankart Repair: Correlation of Results With Postoperative Subscapularis Function. The American journal of sports medicine 2005; 33: 1458-1462

10 Südkamp NP, Stolle S, Bock A, Leupold H, Fournier $C$ von. Die Revision nach initial arthroskopischer oder offener Schulterstabilisierung. Arthroskopie 2000; 13: 229-231

${ }^{11}$ Babcock HM, Carroll C, Matava M, L'Ecuyer P, Fraser V. Surgical site infections after arthroscopy: Outbreak investigation and case control study. Arthroscopy: The Journal of Arthroscopic \& Related Surgery 2003; 19: 172-181

12 Patte D. Classification of rotator cuff lesions. Clinical orthopaedics and related research 1990: 81-86

${ }^{13}$ Ellman H. Diagnosis and treatment of incomplete rotator cuff tears. Clinical orthopaedics and related research 1990; 254: 64-74

14 Ma CB, Comerford L, Wilson J, Puttlitz CM Biomechanical evaluation of arthroscopic rotator cuff repairs: double-row compared with single-row fixation. The Journal of bone and joint surgery 2006; 88: 403-410

${ }_{15}$ Smith CD, Alexander S, Hill AM, et al. A biomechanical comparison of single and double-row fixation in arthroscopic rotator cuff repair. The Journal of bone and joint surgery 2006; 88: 2425-2431

16 Brady PC, Arrigoni P, Burkhart SS. Evaluation of Residual Rotator Cuff Defects After In Vivo Single- Versus Double-Row Rotator Cuff Repairs. Arthroscopy: The Journal of Arthroscopic \& Related Surgery 2006; 22: 1070 1075

17 Mazzocca AD, Millett PJ, Guanche CA, Santangelo SA, Arciero RA. Arthroscopic SingleRow Versus Double-Row Suture Anchor Rotator Cuff Repair. The American journal of sports medicine 2005; 33: 1861-1868
18 Kim DH, ElAttrache NS, Tibone JE, et al. Biomechanical Comparison of a Single-Row Versus Double-Row Suture Anchor Technique for Rotator Cuff Repair. The American journal of sports medicine 2006; 34: 407414

${ }^{19}$ Meier SW, Meier JD. The Effect of DoubleRow Fixation on Initial Repair Strength in Rotator Cuff Repair: A Biomechanical Study. Arthroscopy: The Journal of Arthroscopic \& Related Surgery 2006;22:1168-1173

20 Tuoheti Y, Itoi E, Yamamoto N, et al. Contact Area, Contact Pressure, and Pressure Patterns of the Tendon-Bone Interface After Rotator Cuff Repair. The American journal of sports medicine 2005;33:1869-1874

${ }^{21}$ Anderson K, Boothby M, Aschenbrener D, Holsbeeck M van. Outcome and Structural Integrity After Arthroscopic Rotator Cuff Repair Using 2 Rows of Fixation: Minimum 2Year Follow-Up. The American journal of sports medicine 2006; 34: 1899-1905

22 Ogon P, Ogon M, Jager A. Technical note: The quadrant technique for arthroscopic treatment of rotator cuff calcifications. Arthroscopy: The Journal of Arthroscopic \& Related Surgery 2001; 17: E13-E13

23 Berjano P. Complications in arthroscopic shoulder surgery. Arthroscopy: The Journal of Arthroscopic \& Related Surgery 1998; 14 : 785-788

${ }^{24}$ Armstrong, Bolding, Joseph. Septic arthritis following arthroscopy: Clinical syndromes and analysis of risk factors. Arthroscopy: The Journal of Arthroscopic and Related Surgery 1992: 8: 213-223

25 Gächter A. Der Gelenkinfekt. Inform Arzt 1985; 6: 35-43

${ }_{26}$ Mader JT, Wang J, Calhoun JH. Antibiotic Therapy for Musculoskeletal Infections. The Journal of bone and joint surgery 2001; 83 : $1878-1890$

\section{Dr. med. Martin Jaeger \\ Funktionsoberarzt \\ Dr. med. Wolfgang Köstler \\ Leitender Oberarzt}

Prof. Dr. med. Norbert P. Südkamp

Ärztlicher Direktor

Department Orthopädie und

Traumatologie

Universitätsklinikum Freiburg

Hugstetter Straße 55

79106 Freiburg

\section{Dr. med. Peter Ogon}

Sportorthopädische Praxis

Kaiser-Joseph-Straße 261

79098 Freiburg 